\title{
COMMUNICATION
}

\section{Syntheses of cross-linked polymeric superparamagnetic beads with tunable properties}

Cite this: DOI: $10.1039 / x 0 x x 00000 x$

\author{
Lionel Maurizi ${ }^{\mathrm{a}}$, Usawadee Sakulkhu ${ }^{\mathrm{a}}$, Lindsey A. Crowe ${ }^{\mathrm{b}}$, Vanessa Mai Dao ${ }^{\mathrm{a}}$, \\ Nicolas Leclaire ${ }^{\mathrm{a}}$, Jean-Paul Vallée ${ }^{\mathrm{b}}$, Heinrich Hofmann ${ }^{\mathrm{a}}$
}

Received 00th January 2012,

Accepted 00th January 2012

DOI: $10.1039 / \mathrm{x} 0 \mathrm{xx} 00000 \mathrm{x}$

www.rsc.org/

\section{A novel, rapid and reproducible method to synthesize functionalized magnetic beads with an original shape is presented. By coating $\mathrm{Fe}_{2} \mathrm{O}_{3}$ nanoparticles with $\mathrm{PVA}$, it was possible to tune the size, the number of iron oxide nanoparticles encapsulated and the open porosity of silica beads. Moreover, with PVA pre-modification, functionalized nanobeads were obtained.}

Superparamagnetic iron oxide nanoparticles (SPION) have been developed for biomedical applications during the last decades (e.g., as contrast agents for magnetic resonance imaging (MRI) ${ }^{1-3}$, hyperthermia $^{4,5}$ and magnetic cell labeling ${ }^{6}$ ). Today, only the application as a contrast agent for liver imaging is accepted by the national and European medical agencies, and the large potential for applications in molecular imaging, ex vivo blood purification or hyperthermia are still in the development phase. All these new applications require particles with a much more complex coating, enabling a long residence time in blood and lymph systems, the targeting of tumour cells with a high specificity and an easy clearing mechanism and must fulfil the physical needs for imaging and heating.

SPION are not colloidally stable in physiological conditions due to the reduction of electrostatic repulsion ${ }^{7}$, causing nanoparticle aggregation. To enable the dispersion of SPION in aqueous media and at physiologic salt concentrations, further surface modifications are required. Coating with organic polymers ${ }^{8-10}$ or inorganic layers are the main strategies employed. The coating of SPION with a polymer layer such as polyvinyl alcohol (PVA) ${ }^{11}$ can control the size of aggregates and stabilize the SPION in high ionic strength media at a $\mathrm{pH}$ of approximately 7. PVA is often used in bio-applications because of its non-toxicity, variety of molecular weights and good adhesion on metal oxide nanoparticles ${ }^{12,13}$. However, because PVA is only absorbed onto the iron oxide surface, the amount applied is difficult to control after dilution or mixing with cell media, and some desorption of the polymer from SPION surfaces could occur. Another method to stabilize the SPION is to encapsulate them in a silica layer ${ }^{14-16}$. This coating charges the particles negatively and thus stabilizes the coating electrostatically under biomedical conditions ${ }^{17,18}$. The sol-gel process ${ }^{19}$ has been more widely adopted for the preparation of silica-coated magnetic nanoparticles because of its advantages compared with other methods, i.e., the relatively mild reaction condition, low cost, surfactant-free media and ease of production. The sol-gel process reaction occurs in two steps: hydroxylation and condensation ${ }^{20}$. These reactions are affected by several parameters such as the solvent, temperature, type of precursor, concentration of precursors, $\mathrm{pH}$ and agitation ${ }^{21-23}$. Silicacoated SPION are promising and important in the development of magnetic nanoparticles for both fundamental study and technology applications.

To fulfil all these requirements, the size, coating and functionalization must be developed for each application in a very specific manner, which leads to tremendous effort in the development of the core particles as well as the coating. Additionally, the reproducibility of the manufacturing method and its scale up is often a challenging task because the processes are too complex and of low robustness. This could prevent a translation to clinical research in clinics.

Based on our experience in the manufacturing of PVA and silica-coated SPION, we propose a simple, rapid and reproducible method in this communication to synthesize magnetic functionalized beads through the combination of SPION that are first coated with PVA and then encapsulated in silica beads. PVA-SPION@ silica nanohybrids have a raspberry-like shape, which is different from the commonly observed spherical silica beads. Moreover, it is possible to tune the properties of these beads (their size, the number of SPION entrapped, open porosity, biomolecule inside) by adjusting the initial mass ratio $\mathrm{PVA} / \mathrm{Fe}(\mathrm{Ri})$ and by pre-functionalizing the PVA. Systematic characterizations (such as microscopic observations, and diameter and specific surface area measurements) permit us to conclude a cross-linking process of PVA-SPION and silica. The absence of cytotoxicity and the high rate of internalization of these functionalized magnetic beads into various cell types including HeLa cells, RAW cells, macrophages, monocytes and synoviacytes demonstrate their high potential for biomedical applications.

SPION were synthesized following a classical co-precipitation protocol ${ }^{24,25}$. The PVA-SPION were prepared using an equi-volume mixture of SPION and PVA solutions to obtain PVA/Fe mass ratios (Ri) of 0 (water solution, no PVA), 1, 2, 4, 5, 7 and $9^{25}$. 


\section{COMMUNICATION}
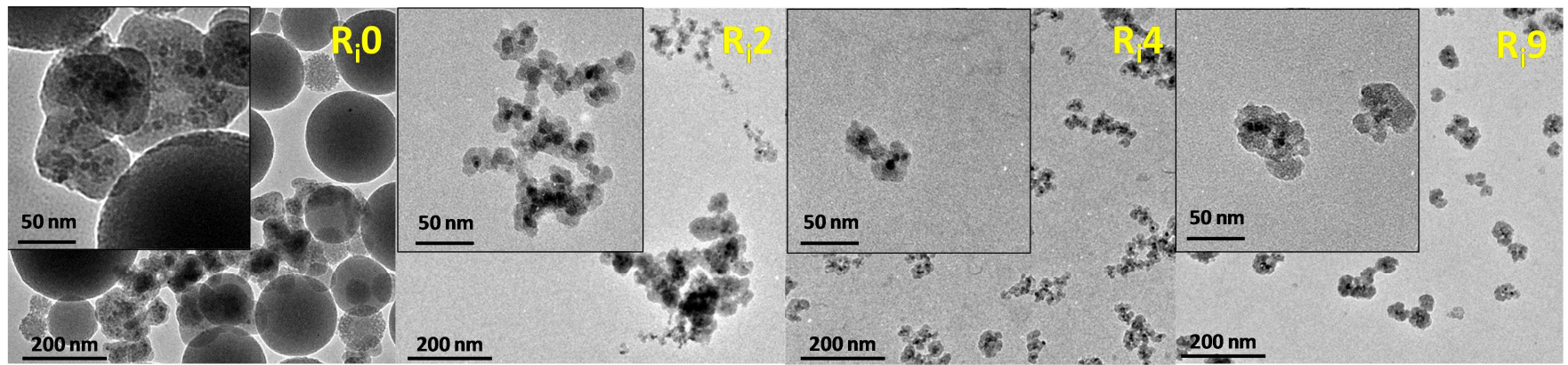

Figure 1: TEM images of PVA-SPION@silica with PVA/Fe mass ratios of Ri0, Ri2, Ri4 and Ri9

Table 1: Characterizations of PVA-SPION@ silica with PVA/Fe ratios ranging from 0 to 9 with or without fluorophore

\begin{tabular}{|c|c|c|c|c|c|c|c|c|c|c|}
\hline $\begin{array}{l}\text { Initial } \mathrm{PVA} / \mathrm{Fe} \\
\text { ratio }\end{array}$ & FITC & $\begin{array}{l}\text { TEM size } \\
\quad(\mathrm{nm})\end{array}$ & $\begin{array}{l}\text { DLS Size } \\
(\mathrm{nm})\end{array}$ & $\begin{array}{c}\text { SPION } \\
\text { /bead }\end{array}$ & $\begin{array}{c}\text { SSA } \\
(\mathrm{m} 2 / \mathrm{g})\end{array}$ & $\begin{array}{r}\text { dBET } \\
(\mathrm{nm})\end{array}$ & $\begin{array}{l}\text { Zeta potential } \\
\qquad(\mathrm{mV})\end{array}$ & $\begin{array}{l}\text { Final } \mathrm{PVA} / \mathrm{Fe} \\
\text { ratio }\left(\mathrm{R}_{\mathrm{f}}\right)\end{array}$ & $\begin{array}{c}\text { Rf } \\
\text { "group" }\end{array}$ & $\begin{array}{c}\kappa \\
\left(\mathrm{m}^{3} \cdot \mathrm{kg}_{\mathrm{Fe} 2 \mathrm{O} 3^{-1}}\right)\end{array}$ \\
\hline $\mathrm{R}_{\mathrm{i}} 0$ & No & nd & $219 \pm 10$ & nd & 5 & 802 & $-40 \pm 2$ & 0 & \multirow{3}{*}{$\operatorname{Rf} \leq 1$} & nd \\
\hline $\mathrm{R}_{\mathrm{i}} 1$ & No & nd & $177 \pm 25$ & nd & 52 & 82 & $-42 \pm 1$ & 0.8 & & nd \\
\hline $\mathrm{R}_{\mathrm{i}} 2$ & No & nd & $135 \pm 11$ & nd & 57 & 75 & $-37 \pm 2$ & 0.8 & & nd \\
\hline $\mathrm{R}_{\mathrm{i}} 4$ & No & $37 \pm 9$ & $80 \pm 11$ & 2.2 & 57 & 75 & $-36 \pm 1$ & 1.1 & \multirow{2}{*}{$1 \leq \mathrm{Rf} \leq 1.5$} & $2.210^{-3}$ \\
\hline $\mathrm{R}_{\mathrm{i}} 5$ & No & $39 \pm 10$ & $86 \pm 9$ & 2.6 & 62 & 69 & $-34 \pm 1$ & 1.4 & & $2.010^{-3}$ \\
\hline $\mathrm{R}_{\mathrm{i}} 7$ & No & $44 \pm 9$ & $92 \pm 8$ & 3.0 & 156 & 27 & $-33 \pm 2$ & 1.9 & \multirow{3}{*}{$\mathbf{R f} \geq \mathbf{2}$} & $2.110^{-3}$ \\
\hline $\mathrm{R}_{\mathrm{i}} 9$ & No & $48 \pm 10$ & $105 \pm 8$ & 4.3 & 122 & 35 & $-36 \pm 2$ & 1.8 & & $2.210^{-3}$ \\
\hline $\mathrm{R}_{\mathrm{i}} 9 *$ & Yes & $46 \pm 10$ & $110 \pm 8$ & 3.8 & 132 & 32 & $-33 \pm 1$ & 1.9 & & $2.110^{-3}$ \\
\hline
\end{tabular}

FITC (\%): mass percentage of A-PVA-FITC added compared with the A-PVA mass already added; TEM size: size measured by counting diameters on TEM images; DLS size: hydrodynamic number weighted diameter; SSA: specific surface area measured by BET, dBET: diameter calculated with SSA values; $\kappa$ :

mass specific susceptibility per $\mathrm{kg} \mathrm{Fe}_{2} \mathrm{O}_{3}$, nd: not determined as a measurement was not possible because of high agglomeration or sedimentation.

Mixtures of PVA were prepared using a 0.9 volume of $12 \mathrm{kDa}$ polyvinyl alcohol (PVA-OH) and a 0.1 volume of $80-140 \mathrm{kDa}$ amino-PVA (A-PVA) copolymer in a mass ratio of $45 / 1$. The obtained PVA-SPION were labeled with the PVA/Fe mass ratio number (Ri). The PVA-SPION Ri9 were also functionalized with fluorescein modified amino-PVA ${ }^{26}$ in a proportion of $10 \%$ of the mass of A-PVA already added (labelled PVA-SPION Ri9*). The 5 $\mathrm{mg}$ of iron of PVA-SPION was mixed with $36 \mathrm{~mL}$ of ethanol and $1.7 \mathrm{mmol}(375 \mu \mathrm{L})$ of tetraethyl orthosilicate (TEOS). Next, $4 \mathrm{~mL}$ of ammonia $28 \%\left(\mathrm{NH}_{4} \mathrm{OH}\right)$ was added to the suspension and was mixed for 90 minutes. The suspensions were then washed 3 times with water and centrifuged. The particles were labelled PVASPION@silica RiX, where X is the PVA/Fe mass ratio used.

The silica beads were characterized using electronic microscopy (morphology and average diameter), dynamic light scattering (hydrodynamic number weighted diameter), BET method (specific surface area, BET diameter), zeta potential, thermogravimetric analyses (TGA: PVA final ratio Rf after reaction) and induced coupled plasma spectroscopy (ICP) and magnetic susceptibility ${ }^{27}$ (number of SPION per silica bead). Mass specific magnetic susceptibilities $\left(\kappa\right.$ in $\left.\mathrm{m}^{3} \cdot \mathrm{kg}^{-1}\right)$ were measured with a Bartington MS3 magneto-susceptometer at $300 \mathrm{~K}$ with a MS2G mono frequency sensor at $(1.3 \mathrm{kHz}) . \mathrm{T}_{2}$ signal loss and quantifiable dUTE (difference ultrashort echo time $)^{28-30}$ Magnetic resonance imaging (MRI) measurements were done on the PVA-SPION@silica Ri9 on a Siemens Magnetom ${ }^{\circledR}$ Trio 3T with nanoparticle concentrations of up to $200 \mu \mathrm{g} / \mathrm{ml}$. The bio-interactions of PVA-SPION@ silica Ri9 and Ri9* were studied using cytotoxicity assays with MTS assays and confocal microscopy on RAW 264.7 cells (mouse leukemic monocyte cell line).
TEM observations of the PVA-SPION@ silica beads (Figure 1) revealed a change of the morphology of the nanoparticles related to the increase of Ri. At Ri0, meaning SPION without PVA, 2 different populations were observed: $100-200 \mathrm{~nm}$ spherical SPION core-free silica nanoparticles and a thin silica shell chain-like structure of silica embedded with SPION. In contrast to the regular spherical shape of silica nanoparticles, the presence of PVA affected the condensation of the silica onto the SPION, leading to an irregular raspberry-like nanoparticle formation. With Ri1 and Ri2, the PVASPION@ silica are inhomogeneous, and again 2 different populations were observed: SPION encapsulated in a sort of raspberry shape of aggregated silica beads and a chain-like structure of PVA-SPION with a thin layer of silica shell. For these 2 ratios, core-free and spherical silica nanoparticles were not observed. At $\mathrm{Ri} \geq 4$, individual PVA-SPION@silica homogenous nanoparticles could be observed. The shape of these beads was still raspberry-like, and the mean diameter increased with the PVA/Fe ratio. Moreover, adding a small amount of A-PVA-FITC did not appear to affect the size or shape of the silica beads at the same PVA/Fe ratio.

The sizes were measured using PVA-SPION@silica TEM images when the samples were homogenous and individual beads were easy to observe, such as for the ratios $\mathrm{Ri}=4,5,7,9$ and $9^{*}$ (Table 1). Even if the standard deviation is high, it can be concluded that an increase of the PVA/Fe mass ratio leads to an increase of the single silica nanoparticle sizes. The hydrodynamic diameters (Table 1) first decrease from Ri 0 to 4 and then increase from Ri 4 to 9 or 9*. As already observed in the TEM images, this result validated the finding that the particles prepared from $\mathrm{PVA} / \mathrm{Fe}$ ratios $\leq 2$ were larger than those prepared from $\mathrm{PVA} / \mathrm{Fe}$ ratios $\geq 4$ and proved that more individual silica beads were present with $\mathrm{Ri} \geq 4$ than with $\mathrm{Ri} \leq 2$. 
This result could be explained by a different degree of aggregation of the initial PVA-SPION. For the ratio Ri0, the hydrodynamic sizes should be the sizes of the SPION-free silica nanoparticles already observed by TEM. For $\mathrm{Ri} \geq 4$, the number of SPION encapsulated in the beads increased from $2.2(\mathrm{Ri}=4)$ to $4(\mathrm{Ri}=9$ or $9 *)$. For lower values of $\mathrm{Ri}$, the determination of the number of SPION/bead was not possible (the agglomerate was too large).

The increase of the specific surface areas (SSA) was related to an increase of the PVA/Fe ratio. For Ri 0, 1 and 2, it was difficult to correlate the SSA to the PCS or TEM sizes because of the heterogeneity of the particles, as indicated by the TEM images. For instance, for the PVA-SPION@silica Ri0, the value of $5 \mathrm{~m}^{2} \cdot \mathrm{g}^{-1}$ provided evidence of the presence of large agglomerated SPION with a thin layer of silica. In contrast, the SSAs of PVASPION@silica Ri1 and Ri2 were larger than expected from the PCS sizes, demonstrating the possible presence of uncoated SPION or very-thin-silica-layer-coated SPION. However, for the ratios 4 and 5 , the SSAs correlated well with the sizes obtained from PCS and TEM images. Finally, for the ratios 7, 9 and 9*, the SSAs was much higher than predicted from the TEM and PCS sizes. An explanation for this finding could be the presence of more open porosity in the PVA-SPION@silica Ri7, Ri9 and Ri9*, which increased the SSAs. The zeta potentials on the different silica particles obtained were not significantly different in the range of -33 to $-42 \mathrm{mV}$. The naked SPION have a saturation magnetization of $63 \mathrm{emu} / \mathrm{g}^{31}$ and a mass specific susceptibility $(\kappa)$ of $1.910^{-3} \mathrm{~m}^{3} \mathrm{~kg}^{-1}$. This is in the same range as the $\kappa$ of the PVA-SPION@silica for $\mathrm{Ri} \geq 4$ proving that the magnetic properties of the iron oxide nanoparticles is not changed by the coating or by the assembly of iron oxide nanoparticles.

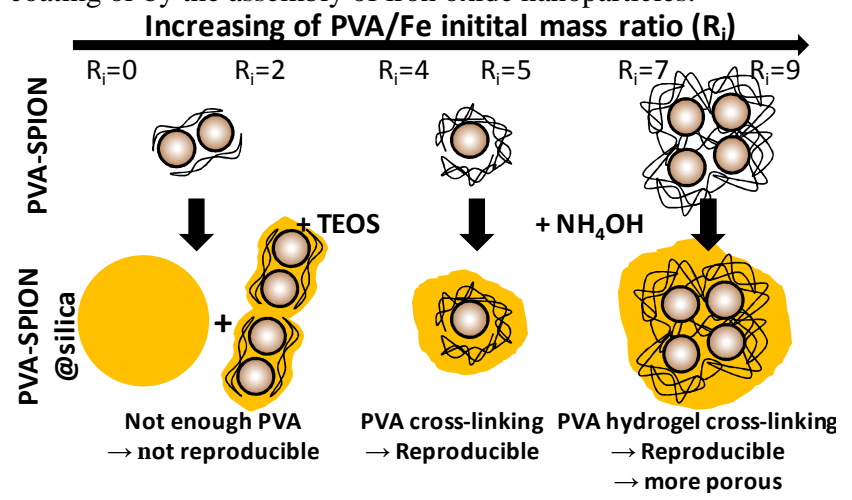

Figure 2: Schematic representation of the proposed PVA-SPION/silica crosslinking mechanism as a function of the PVA/Fe initial mass ratio (Ri). From $\mathrm{Ri} 0$ to $\mathrm{Ri}$, the SPION are not stabilized enough and are aggregated in silica coating lines with silica beads without SPION or agglomerated. For Ri4 and

Ri5, the PVA-SPION are stabilized and cross-linked in a raspberry-like shape; by increasing $\mathrm{Ri}$ to $\mathrm{Ri} 7$ or $\mathrm{Ri} 9$ and $\mathrm{Ri9} 9^{*}$, the silica beads increased in size and became more porous.

Finally, the final PVA/Fe ratios (Rf) in the silica beads measured using TGA could be divided into 3 different groups according to the results in Table 1. First, Rf values less than 1 were measured on the silica particles with Ri0, 1 and 2 . Rf values of approximately 1.5 were observed for the initial PVA-SPION PVA/Fe ratios of Ri4 and Ri5. Silica particles with Ri7, 9 and $9^{*}$ exhibited a final PVA mass ratio $\mathrm{Rf}$ of approximately 2 . The final PVA/Fe mass ratio (Rf) appears to be the most important parameter affecting the chemical properties of the beads. Three different Rf were also observed in previous work (Chastellain et al. ${ }^{32}$ ). It was suggested in this study that below Rf1, the SPION were not stabilized enough by PVA; from Rf1 to Rf2, single SPION were coated and stabilized and above Rf2, the PVA-SPION formed a hydrogel with several SPION inside.
This assumption was also verified during this work (Figure 2). In fact, when the Rf final ratio is below 1, the silica particles obtained have inhomogeneous shapes with large sizes and SPION aligned in a thin silica shell, demonstrating that PVA was not sufficient to stabilize the SPION. When the final PVA/Fe Rf ratio was above 1, the particles obtained were homogenous and reproducibly synthesized, demonstrating that PVA-stabilized SPION helped in the formation of a silica layer, which increased with the quantity of PVA used. The increase of the number of SPION/beads linked to the increasing $\mathrm{Ri}$ could support this theory. The original shape observed could be a cross-linking of the PVA-SPION and the silica because the $\mathrm{OH}$ groups on both SPION and PVA were able to condensate silica. This conclusion is supported by the higher open porosity observed for an $\mathrm{Rf}$ value of approximately 2, which is most likely evidence of the cross-linking of silica on the PVA-SPION hydrogel. Finally, the presence of FITC-functionalized amino-PVA on the SPION before silica coating does not affect the PVA-SPION@silica Ri9* characteristics. Signal loss, due to $\mathrm{T}_{2}$ effects clearly shows detection of the SPION even at very low concentrations (Figure 3-a). At moderate concentrations ( $\geq 25 \mu \mathrm{g} / \mathrm{mL}$ on Figure $3 \mathrm{a}$ top), the signal is zero and therefore we have good contrast, but lose concentration information. The previously proposed dUTE method gives quantifiable signal and supressed image background (Figure 3a, lower image). MRI shows easy detection and high sensitivity as well as quantifiable imaging dependent on concentration in line with previously studied contrast agents.

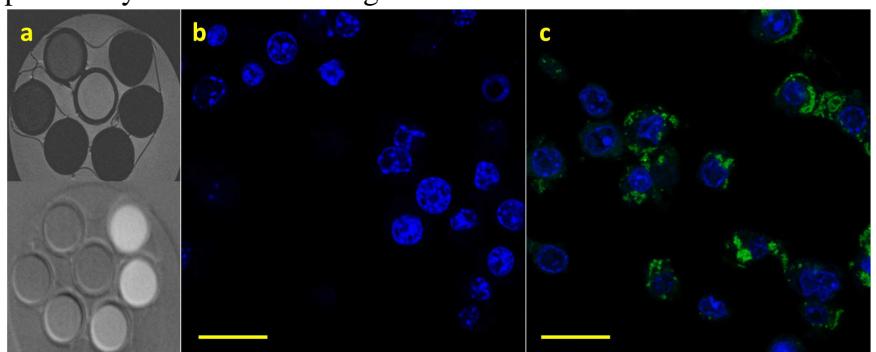

Figure 3: a: $\mathrm{T}_{2}$ signal loss (upper) and quantifiable dUTE (lower) images of PVA-SPION@ silica Ri9: centre: water; concentration anti-clockwise from top left: $6.25,12.5,25,50,100,200 \mu \mathrm{g} / \mathrm{ml} ; \mathrm{b}$ and $\mathrm{c}$ : Confocal imaging of RAW 264.7 cells incubated with: b) 0.4 mg PVA-SPION@ silica Ri9 per mL and c) 0.4 mg PVA-SPION@ silica Ri9* per mL. Blue colour: nuclei labelled with DAPI. Green colour: FITC labelled nanoparticles. Scale-bar: $20 \mu \mathrm{m}$

Regarding bio-interactions, PVA-SPION@silica Ri9 and Ri9* did not exhibit any cytotoxicity $24 \mathrm{~h}$ after incubation. The internalizations of these particles into macrophage cells were observed by confocal microscopy (Figure $3 \mathrm{~b}$ and c). For PVASPION@silica Ri9, only the blue-labeled nuclei were detected without specific fluorescence at FITC wavelength. In contrast, in the presence of fluorescently labeled silica particles (PVASPION@silica Ri9*), the green color, which represented PVASPION@silica Ri9*, was observed to be surrounding the nuclei, revealing the presence of these particles inside the cells. These first biological experiments demonstrated that the cross-linked silicaPVA-SPION did not exhibit any cytotoxicity.

In conclusion, it is possible to prepare new magnetic silica bead nanomaterials and to tune their sizes and the number of nano-objects inside their matrix by changing the initial mass ratio of the polymer and nanoparticles from $\mathrm{Ri}=0$ to 9 . The raspberry-like shape of the resulting nanohybrids is different from the usually spherical silica particles. This difference can be explained by a cross-linking process that occurs between the PVA and the silica during the hydrolysis condensation reaction, which leads to more open porosity when $\mathrm{Ri} \geq 7$. By pre-functionalizing the polymer before SPION coating, fluorescent magnetic silica beads were easily obtained. Using this PVA stabilization method, other biomolecules could be incorporated 
in magnetic beads, which coupled with the rough surface, higher 25 . open porosity and first bio-characterizations, could be valuable for biomedical applications. Furthermore, the replacement of TEOS with an amine-functionalized precursor could lead to particles with controlled zeta potentials, starting from very negative potentials using TEOS to highly positive potentials using APTES for the crosslinking of PVA. The developed methods permit the manufacturing of unique building blocks that are ready for further functionalization by biomolecules, and a concept that significantly improves the reproducibility of the nanoparticle manufacturing.

Notes and references

a Powder Technology Laboratory, Ecole Polytechnique Federale de Lausanne (EPFL), 1015 Lausanne, Switzerland, lionelmaurizi@gmail.com

b Department of Radiology, University of Geneva and Geneva University Hospital, 1211 Geneva 14, Switzerland

Electronic Supplementary Information (ESI) available: [details of any supplementary information available should be included here]. See DOI: 10.1039/c000000x/

1. U. I. Tromsdorf, O. T. Bruns, S. C. Salmen, U. Beisiegel, and H. Weller, Nano Lett, 2009, 9, 4434-40.

2. E. A. Schultz-Sikma, H. M. Joshi, Q. Ma, K. W. MacRenaris, A. L. Eckermann, V. P. Dravid, and T. J. Meade, Chem. Mater., 2011, 23, 26572664

3. J. Boudon, J. Paris, Y. Bernhard, E. Popova, R. A. Decreau, and N. Millot, Chem. Commun., 2013, 49, 7394-7396.

4. K. Hayashi, K. Ono, H. Suzuki, M. Sawada, M. Moriya, W. Sakamoto, and T. Yogo, Chem. Mater., 2010, 22, 3768-3772.

5. K. Yan, P. Li, H. Zhu, Y. Zhou, J. Ding, J. Shen, Z. Li, Z. Xu, and P. K. Chu, RSC Adv., 2013, 3, 10598-10618.

6. M. J. Pittet, F. K. Swirski, F. Reynolds, L. Josephson, and R. Weissleder, Nat. Protoc., 2006, 1, 73-79.

7. L. Maurizi, F. Bouyer, J. Paris, F. Demoisson, L. Saviot, and N. Millot, Chem. Commun., 2011, 47, 11706-11708.

8. $\quad$ L. Maurizi, H. Bisht, F. Bouyer, and N. Millot, Langmuir, 2009, 25, 88578859 .

9. B. Basly, D. Felder-Flesch, P. Perriat, C. Billotey, J. Taleb, G. Pourroy, and S. Begin-Colin, Chem. Commun., 2010, 46, 985-987.

10. K. M. L. Taylor-Pashow, J. D. Rocca, R. C. Huxford, and W. Lin, Chem. Commun., 2010, 46, 5832-5849.

11. C. Leduc, S. Si, J. Gautier, M. Soto-Ribeiro, B. Wehrle-Haller, A. Gautreau, G. Giannone, L. Cognet, and B. Lounis, Nano Lett., 2013, 13, 1489-1494.

12. K. Schulze, A. Koch, A. Petri-Fink, B. Steitz, S. Kamau, M. Hottiger, M. Hilbe, L. Vaughan, M. Hofmann, H. Hofmann, and B. von Rechenberg, $J$. Nanosci. Nanotechnol., 2006, 6, 2829-2840.

13. T. Valdés-Solís, A. F. Rebolledo, M. Sevilla, P. Valle-Vigón, O. BomatíMiguel, A. B. Fuertes, and P. Tartaj, Chem. Mater., 2009, 21, 1806-1814.

14. T. J. Brunner, P. Wick, P. Manser, P. Spohn, R. N. Grass, L. K. Limbach, A. Bruinink, and W. J. Stark, Environ. Sci. Technol., 2006, 40, 4374-4381.

15. S.-H. Wu, Y. Hung, and C.-Y. Mou, Chem. Commun., 2011, 47, 9972-9985.

16. O. V. Kharissova, B. I. Kharisov, V. M. Jiménez-Pérez, B. M. Flores, and U. O. Méndez, RSC Adv., 2013, 3, 22648-22682.

17. K. L. Young, A. W. Scott, L. Hao, S. E. Mirkin, G. Liu, and C. A. Mirkin, Nano Lett., 2012, 12, 3867-3871.

18. N. Ž. Knežević, E. Ruiz-Hernández, W. E. Hennink, and M. Vallet-Regí, RSC Adv., 2013, 3, 9584-9593.

19. W. Stober, A. Fink, and E. Bohn, J. Colloid Interface Sci., 1968, 26, 62-69.

20. B. Arkles, J. R. Steinmetz, J. Zazyczny, and P. Mehta, J. Adhes. Sci. Technol., 1992, 6, 193-206.

21. Y. H. Deng, C. C. Wang, J. H. Hu, W. L. Yang, and S. K. Fu, Colloids Surf. Physicochem. Eng. Asp., 2005, 262, 87-93.

22. D. K. Yi, S. T. Selvan, S. S. Lee, G. C. Papaefthymiou, D. Kundaliya, and J. Y. Ying, J. Am. Chem. Soc., 2005, 127, 4990-4991.

23. C. W. Lu, Y. Hung, J. K. Hsiao, M. Yao, T. H. Chung, Y. S. Lin, S. H. Wu, S. C. Hsu, H. M. Liu, C. Y. Mou, C. S. Yang, D. M. Huang, and Y. C. Chen, Nano Lett., 2007, 7, 149-154.

24. R. Massart, E. Dubois, V. Cabuil, and E. Hasmonay, J. Magn. Magn. Mater., $1995,149,1-5$
25. A. Petri-Fink, M. Chastellain, L. Juillerat-Jeanneret, A. Ferrari, and H. Hofmann, Biomaterials, 2005, 26, 2685-2694.

26. J. Salaklang, B. Steitz, A. Finka, C. P. O. Neil, M. Moniatte, A. J. V. der Vlies, T. D. Giorgio, H. Hofmann, J. A. Hubbell, and A. Petri-Fink*, Angew Chem Int Ed, 2008, 47, 7857-7860.

27. L. Maurizi, U. Sakulkhu, A. Gramoun, J.-P. Vallee, and H. Hofmann, Analyst, 2013.

28. L. A. Crowe, F. Ris, S. Nielles-Vallespin, P. Speier, S. Masson, M. Armanet, P. Morel, C. Toso, D. Bosco, T. Berney, and J.-P. Vallee, Am. J. Transplant. Off. J. Am. Soc. Transplant. Am. Soc. Transpl. Surg., 2011, 11, 1158-1168.

29. L. A. Crowe, F. Tobalem, A. Gramoun, B. M. A. Delattre, K. Grosdemange, J. Salaklang, A. Redjem, A. Petri-Fink, H. Hofmann, and J.-P. Vallée, Magn. Reson. Med. Off. J. Soc. Magn. Reson. Med. Soc. Magn. Reson. Med., 2012, 68, 1544-1552.

30. S. Nielles-Vallespin, M.-A. Weber, M. Bock, A. Bongers, P. Speier, S. E. Combs, J. Wöhrle, F. Lehmann-Horn, M. Essig, and L. R. Schad, Magn. Reson. Med., 2007, 57, 74-81.

31. A. Petri-Fink and H. Hofmann, IEEE Trans. Nanobioscience, 2007, 6, 289-297.

32. A. Chastellain, A. Petri, and H. Hofmann, J. Colloid Interface Sci., 2004, 278, 353-360. 